\title{
Gold Recovery from Gold Bearing Materials Using Bio-Diesel, Vegetable Oils and Coal
}

\author{
Anderson Mlaki, Jamidu Katima, Henry Kimweri \\ Department of Chemical and Mining Engineering, College of Engineering and Technology, \\ University of Dar es Salaam, Dar es Salaam, Tanzania \\ E-mail:amlaki@udsm.ac.tz,jamidu_katima@yahoo.co.uk,teganikim@gmail.com \\ Received March 14, 2011; revised March 30, 2011; accepted April 25, 2011
}

\begin{abstract}
The present work is focused on the performance of three types of coal-oil agglomerates on recovery of liberated gold from gold bearing materials. Pre-formed agglomerates developed using bio-diesel, castor oil and mineral diesel as liquid hydrophobic phases were used in the study. The influence of the type of liquid hydrophobic phase on the degree of gold recovery and the effect of factors such as gold particle concentration, viscosity, agglomerate size, agglomerate/ore ratios and particle penetration into agglomerates have been studied as a function of time. It is shown that gold recovery rate can be increased by an increase in agglomerate loading, surface area and the viscosity of the hydrophobic phase and high recoveries are attained up to $98.5 \%$. Increase in concentration of gold particles per unit volume of slurry increased attachment rate but did not change the final recoveries attained. It is shown that gold particle penetration occurs mainly in the coarse agglomerates if contact is prolonged beyond 60 minutes. Examination of sections of gold-loaded agglomerates under reflected light microscope showed gold and some silicate particles penetrated in few cracked agglomerates and only gold particles were observed inside the uncracked agglomerates suggesting the possibility of gold selectivity during particles penetration. It was shown that the increase in gold recovery attained at prolonged contact time is due to both gold penetration and oleophilic attachment.
\end{abstract}

Keywords: Pre-Formed Agglomerates, Selective Gold Penetration, Oleophilic Attachment

\section{Introduction}

Many researchers in the area of extractive metallurgy and chemical engineering have reported the possibility of gold recovery by coal-gold-oil agglomeration method, which is a potential alternative to the mercury amalgamation. It is widely accepted that gold surface covered by a layer of hydrocarbon molecule is hydrophobic and hence capable of migrating from gold bearing slurry to agglomerates [1].

Although there are several methods commonly used to extract gold from its ores, the selection of a particular method highly depend on the mineralogical characteristics, commercial viability [2] and environmental friendliness. The hydrophobic recovery of gold using agglomerates is environmentally seen as the best alternative to mercury amalgamation in small scale mining [3].

The positive results from previous research works on coal-oil-gold agglomeration [4-11] and the promising environmental and technical viabilities of the process
[12] have raised the need for adoption of this method in small scale mining in Tanzania where the hazardous use of mercury is still dominant [13], and was the motivation for doing this research.

This work addresses some areas that have hindered the development of this method in small scale mining in Tanzania. The sophistication of the operation in terms of types of hydrophobic materials used and the handling of the effective variables $[8,9,10]$ compared to mercury amalgamation has been addressed.

As an attempt to bring the gold recovery method to small scale miners levels in Tanzania, this research focused on the use of pre-formed agglomerates with parameters such as oil/coal ratio and coal particle size already optimized during agglomerate formation.

Using the standard pre-formed agglomerates it was possible to achieve high gold recoveries.

Three types of preformed agglomerates developed using bio-diesel, castor oil, petroleum diesel and bituminous coal from Kiwira coal deposit were avai- 
lable for the contact experiments. The performance of the agglomerates is presented in terms of gold recoveries.

The selection of the liquid hydrophobic phases used in formation of the agglomerates was based mainly on availability and environmental friendliness. Bio-diesel was considered environmentally friendly and could be produced using locally available oils, the particular biodiesel which was used in the experiments was produced from palm oil and methanol at controlled conditions at the Department of Chemical and Mining Engineering (CME), College of Engineering and Technology UDSM. Castor oil was selected for the experiments as non edible vegetable oil available locally. Small scale miners could be encouraged to plant castor plants for easy production of the oil. Petroleum diesel is mentioned in a number of previous reports and was used as a reference oil phase. Bio-diesel and castor oil agglomerates were tested here for the first time and proved very effective compared to previously tested liquid hydrophobic phases in the ongoing researches on recovery of gold from ores using hydrophobic and oleophilic properties.

\section{Experimental}

\subsection{Materials}

\section{a) Agglomerate samples}

Three sets of samples of pre-formed agglomerates, developed from three different types of liquid hydrophobic phases, castor oil, bio-diesel and mineral/petroleum diesel using bituminous coal from Kiwira were used. The agglomerates were prepared at oil/coal ratio 0.3 , coal particle size of -110 microns and agitation time of 20 minute at $1000 \mathrm{rpm}$ as established from the previous work done and published by the author [14].

b) Gold bearing materials

The gold bearing materials used in the study contained classified fine quartz (100\% - 150\% microns) and metallic gold powder minus 150 microns originating from small scale mining areas in Chunya, Mbeya Region Tanzania.

c) Reagents

Potassium Amyl Xanthate (KAX) commercial yellow pellet form-water soluble, Hydrochloric acid ( $\mathrm{HCl}$ ) and Nitric acid $\left(\mathrm{HNO}_{3}\right)$.

\subsection{Methods}

\section{Gold attachment tests}

The Gold attachment tests were carried out in a 1000 $\mathrm{ml}$ beaker equipped with two baffles $180^{\circ}$ apart. The contents stirred at 700 rpm by a four-bladed stainless steel impeller and 20\% solids. The procedure involved wetting $100 \mathrm{~g}$ of gold bearing material with de-ionized water, adding potassium amyl xanthate collector and agitating for 5 minutes, adding specific amount of agglomerate sample and stirring for a given time. The mixture was screened of on a $0.325 \mathrm{~mm}$ sieve with wash water to separate gold-loaded agglomerates from the pulp. Drying loaded agglomerates in open air for 6 hrs and weighing them followed by assessing the recovery of gold by analyzing the amount of gold attached to agglomerates. Gold analysis was done by roasting the dry agglomerates to ash at $500^{\circ} \mathrm{C}$ in a muffle furnace then treating the ash with aquaregia to dissolve gold. The amount of gold in the diluted gold solution was determined by atomic absorption spectrophotometry.

All the tests were carried out on the basis of liquid hydrophobic phase used in forming the agglomerates (Bio-diesel, Castor oils, Petrol-diesel). Optimum recoveries, agitation time and agglomerate loadings were established.

\section{Results and Discussion}

\subsection{Effect of oil type on the Performance of Agglomerates}

Three types of preformed agglomerates made from castor oil, bio-diesel and petroleum-diesel respectively were brought in contact with gold bearing material containing $1 \%$ gold for 60 minutes as described in section 2.2. The results were evaluated in terms of percentage gold recoveries.

At agglomerate loading of 0.4 , the castor oil agglomerates attained gold recovery of $98.5 \%$ as shown in Figure 1. The performances of bio-diesel and petroleum diesel agglomerates were similar and both attained highest recovery of $93.5 \%$.

Higher gold recoveries were attained by castor oil agglomerates with viscosity of about $891 \mathrm{cP}$ at $25^{\circ} \mathrm{C}$ as

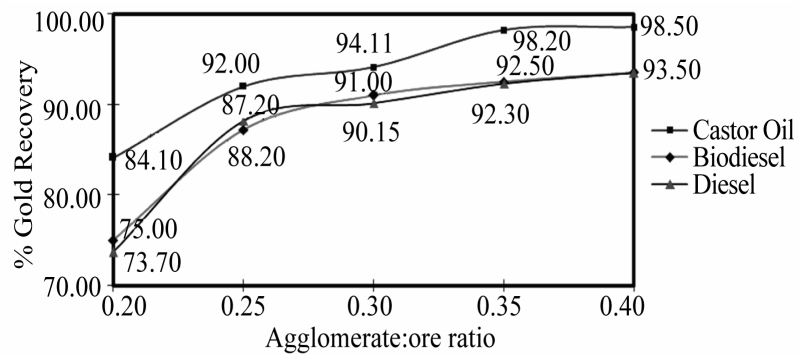

Figure 1. Effect of oil type on the performance of agglomerates at different agglomarate/ore ratios. (Contact time of 60 minutes, agitation speed of $700 \mathrm{rpm}$ ). 
compared to bio-diesel (77.0 cP), and petroleum-diesel $(76.2 \mathrm{cP})$. This was attributed to the formation of more stable agglomerates which stabilized the available adsorption area for gold particles suggesting increased adhesion due to higher viscosity. This is consistent with literature according to Elblbesy [15].

\subsection{Effect of Agglomerate Loading Using Recovery/Time Relationship}

Figures 2(a)-(c) show gold recoveries plotted against contact time at different agglomerate loading. The experiments were conducted in order to determine completion time for gold adsorption and optimum recoveries. Three samples of different agglomerate/ore ratios of $0.25,0.3$, and 0.4 were each divided into five equal parts making a total of 15 sub-samples. Each set of five were treated for gold recovery at contact times of; 30 minutes; 60 minutes; 90 minutes; 120 minutes and 150 minutes respectively to compare performance between short and long contact time.

The results showed that low agglomerate/ore ratios required prolonged contact time for completion of gold recovery. High agglomerate loading lowered the time taken to attain optimum gold recovery as shown in Figures 2(a)-(c). It is also shown that, high agglomerate-ore ratios increase the rate at which gold particles are recovered per unit time. The available surface area for particle attachment which varied according to agglomerate loading was considered as the main factor affecting completion time, optimum recoveries and recovery rate.

\subsection{Effect of Viscosity of the Liquid Hydrophobic Phase on the Rate of Gold Recovery}

In order to show the effect of viscosity on the rate of gold recovery, the recoveries were plotted against time

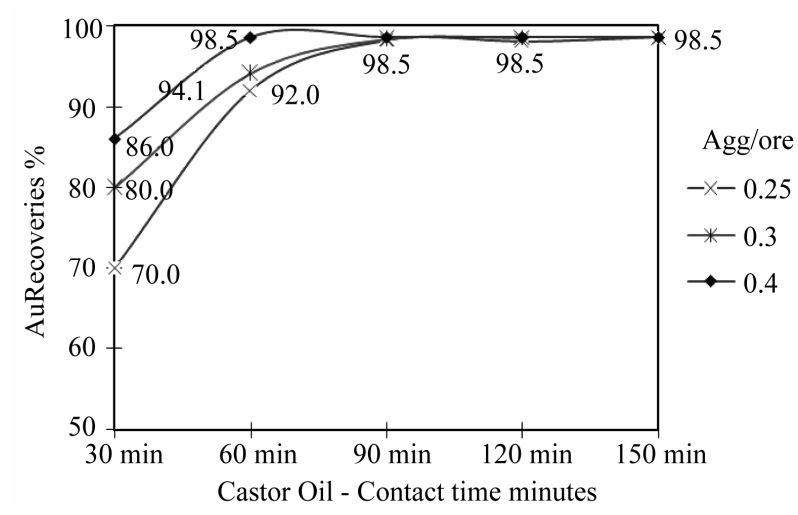

(a)

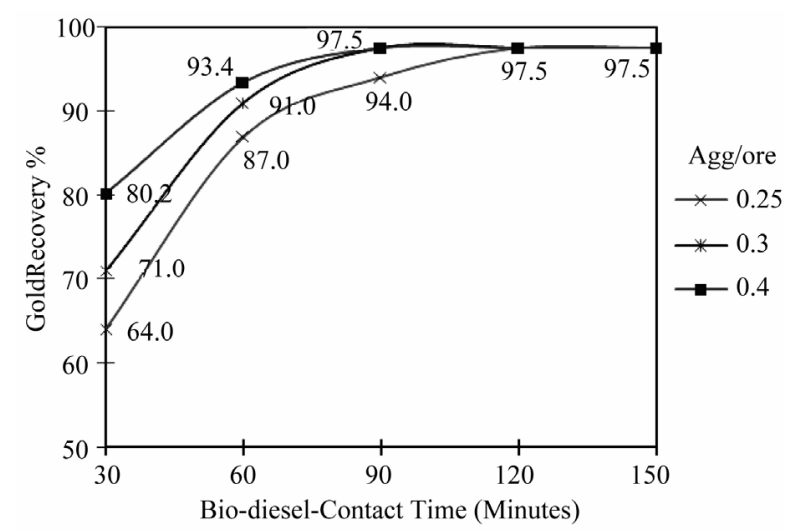

(b)

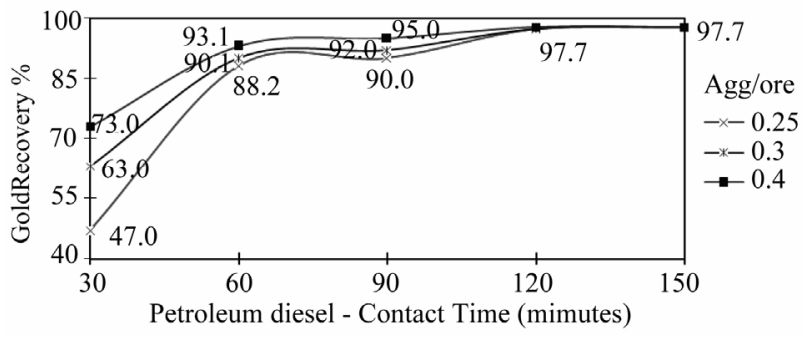

(c)

Figure 2. Recovery-Time plots showing completion of gold recovery for castor oil, bio-diesel and petroleum diesel agglomerates (700 rpm, $20 \%$ solids pulp density)".

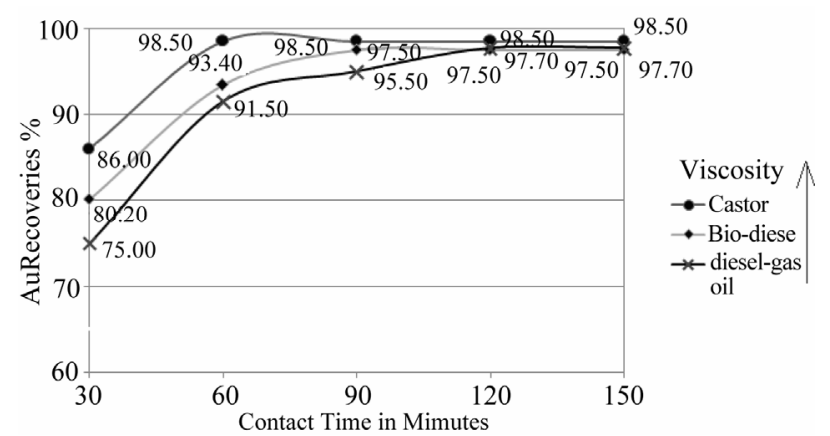

Figure 3. Recovery/Time Curves showing the effect of viscosity on recovery rate at constant aggl/ore ratio of 0.4 .

at constant agglomerate loading as shown in Figure 3. It was noted that at any point on the curves, the higher viscosity agglomerates had higher recoveries than the lower viscosity agglomerates, indicating an increase in recovery rate with increase in viscosity of hydrophobic phase. After 120 minutes the gradients of the curves were the same indicating possible completion of gold removal. It was suggested that higher viscosity reduced shear on agglomerates and increased stability of agglomerate surface area available for adsorption of gold particles.

Generally, the results showed that increase in viscosity of the hydrophobic phase increases not only the 
amount of gold recovered, but also the gold recovery rates as shown in Figure 3.

\subsection{Effect of Gold Particle Concentration on Gold Attachment Rate}

High gold recovery rates were observed at the initial stages of contact and decreased with increase in contact time regardless of the type of liquid hydrophobic phase used. This was explained as due to higher chances of gold-agglomerate contact at high concentration of free gold particles per unit volume in the slurry. Concentration was high during the initial stages of adsorption and decreased with increase in contact time.

According to the recovery-time plots, the gradients of recovery/time curves decreased gradually to hori- zontal after 90 minutes (Figures 2(a)-(c)) indicating completion of gold transfer from the slurry to agglomerates. The gold transfer process suggested a first order reaction with high concentration indicating high driving force in agreement with the mass balance equation at completion of gold removal that; $\mathrm{C}_{\text {in }}=\mathrm{C}_{\text {out }}$ where ' $\mathrm{C}_{\text {in }}$ ' represent gold in slurry and ' $\mathrm{C}_{\text {out }}$ ' is the gold attached to agglomerates.

Images of the ore samples taken under reflected light microscope were used to show the particles structure at different contact time and showing the gradual changes in gold concentration in the slurry ' $\mathrm{C}_{\mathrm{in}}$ '.

The images showed a decrease in gold particles available in the slurry with increase in contact time as shown in Figures 4-6 when the ore samples were taken from the slurry at different contact times, filtered, air dried and observed under a microscope.

It is noted in Figures 4-6, that after 60 minutes contact time, gold particles could still be observed in the ore sample indicating that the transfer of gold particles to agglomerates could not be completed due to high number of particles available. The results have shown that the concentration of gold particles in the slurry has

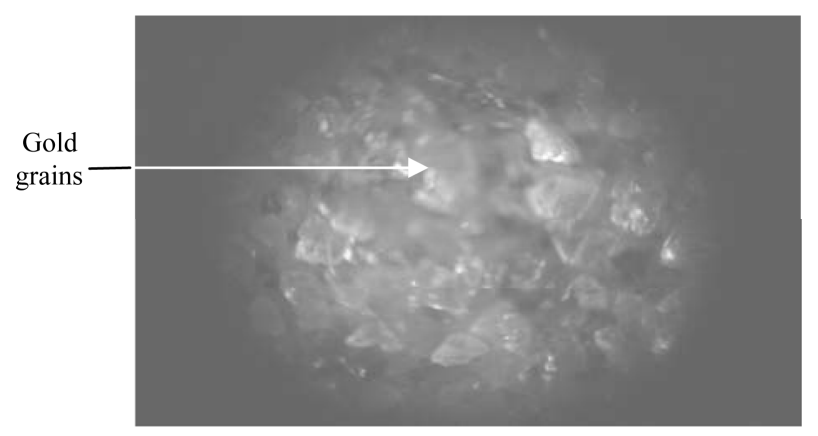

Figure 4. Gold bearing material showing gold particles before the start of gold contact with agglomerates. Magnification $\times 10$.

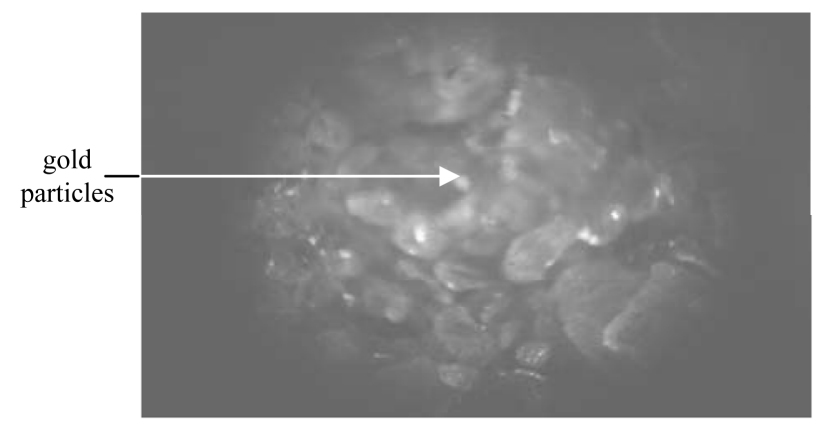

Figure 5. Image showing gold particles in after 60 minutes contact with agglomerates. Magnification $\times 10$.

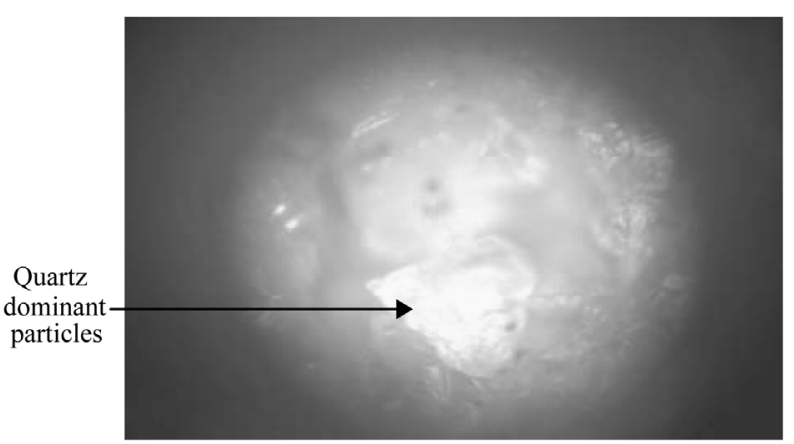

Figure 6. Image, showing absence of gold particles after $\mathbf{9 0}$ minutes contact time due to completion of gold removal. Magnification $\times 15$.

an influence on the completion time for gold transfer from slurry to agglomerates and hence affects gold recovery rate.

\subsection{Effect of Agglomerate Size on the Amount of Recoverable Gold}

The chart in Figure 5 shows the performance of classified agglomerates comprising coarse and fine size fractions. Basing on the results it was possible to draw certain inferences on the effect of agglomerate size on gold recovery at prolonged contact time.

Coarse agglomerates of size $(-2 \mathrm{~mm}+1.5 \mathrm{~mm})$ and $(-1.5+1 \mathrm{~mm})$ and fine agglomerates of size $(-1 \mathrm{~mm}+$ $0.5 \mathrm{~mm})$ and $(-0.5 \mathrm{~mm}+0.325 \mathrm{~mm})$, were used in the tests. Gold recoveries were attained from each agglomerate size at different contact time.

At 60 minutes contact time, the fine agglomerate fractions $(+0.5-1.0 \mathrm{~mm})$ and $(-0.5 \mathrm{~mm})$ attained respective recoveries of $96.10 \%$ and $96.25 \%$ while the coarser fractions $(+1-1.5)$ and $(+1.5-2.0)$ attained lower recoveries $92.26 \%$ and $92.56 \%$ respectively. It is clear that the differences in recovery between the fine and the coarse were due to greater surface area per unit weight of the finer agglomerates.

At 90 minutes contact time, all agglomerate sizes had 


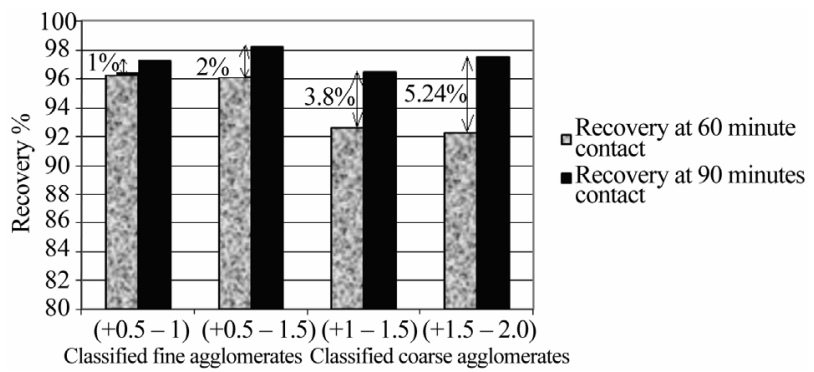

Figure 7. A chart showing the recoveries attained by different agglomerate sizes.

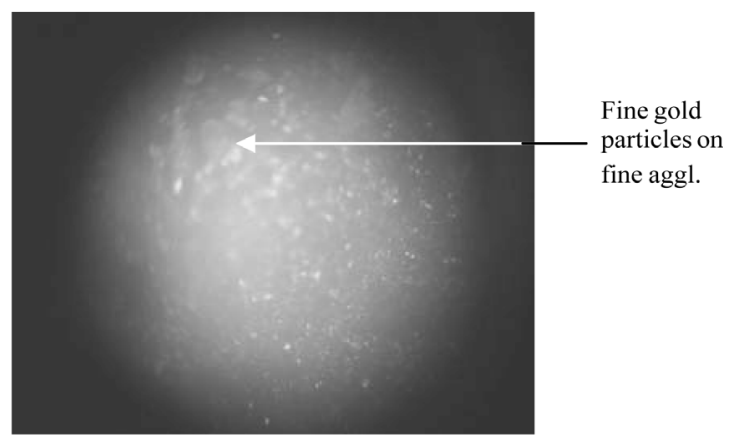

(a)

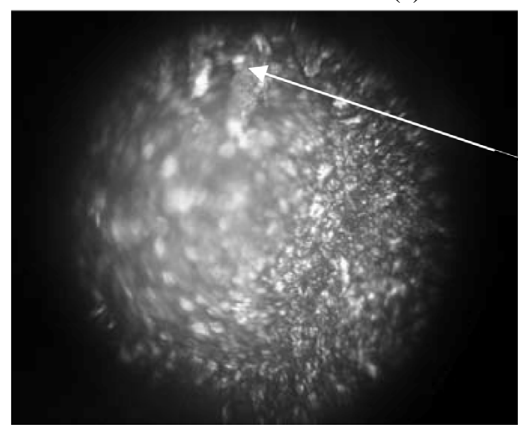

Coarse gold particle on the surface of coarse aggl. Note: Particles shape corresponds to original recovery method

(b)

Figure 8. Images of loaded agglomerates showing gold adsorption on the surface of agglomerate. (a) Magnification $\times$ 10 and (b) Magnification $\times 25$. Note: The gold particles used originated from burning of gold-mercury amalgam and had variable shapes

attained additional recoveries though at different magnitudes. It was noted that the coarsest agglomerates had higher additional recovery of $5.24 \%$ compared to the increase in the finest agglomerates of only $1 \%$. This difference was explained as due to interstitial penetration of fine gold particles into the coarse agglomerates caused by probably capillarity and/or external forces resulting from inter-particle collision and shearing forces caused by impellers and baffles [16].

\subsection{Gold Loaded Agglomerates Examined Using Reflected Light Microscope}

Figure 8(a) and (b), are images of loaded agglomerate

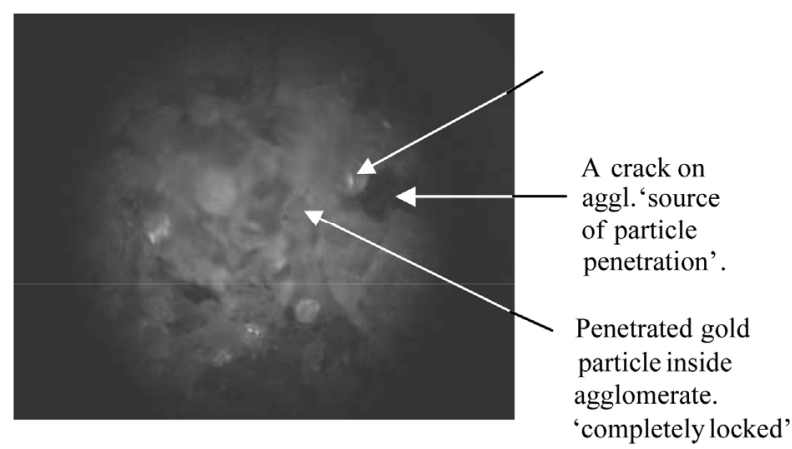

Figure 9. Image of loaded sliced coarse agglomerate of size $+1.5-2 \mathrm{~mm}$ at magnification $\times 15$.

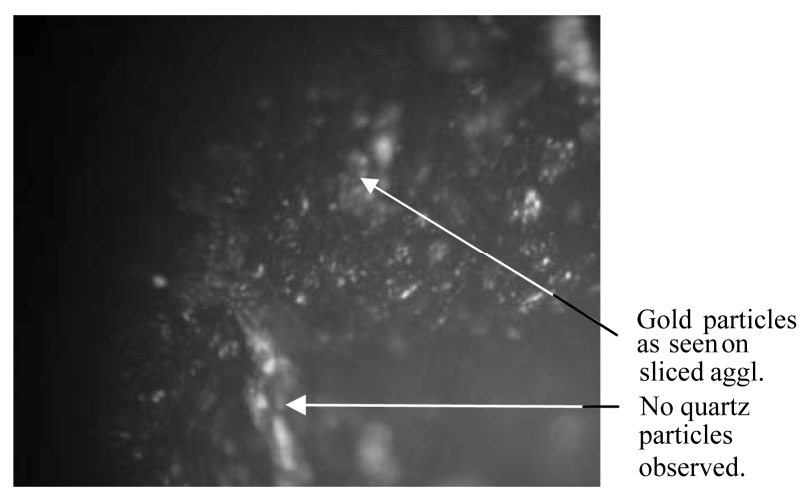

Figure 10. Gold inside sliced coarse agglomerate without cracks $\times 50$ magnification.

after 60 minutes contact time showing gold particles attachment on the surface of fine and coarse agglomerate respectively. According to the images, mainly gold particles were observed on the surface of agglomerates indicating high selectivity during attachment at $60 \mathrm{~min}$ utes contact time. Washing the loaded agglomerates during screening improved visibility of the attached gold under reflected light as shown in Figure 10(b).

Figures 9 and $\mathbf{1 0}$ show images of sliced sections of loaded agglomerates after 90 minutes contact. It was noted that fine gold particles were penetrated and some of them were completely locked in the agglomerate, indicating interstitial penetration.

In Figures 9 some penetrated gold particles were situated very close to the crack, indicating the possibility of gold penetration through the cracks. Some quartz particles could be observed in the cracked agglomerates indicating recovery dilution due to presence of cracks.

Figures 10 shows the section of uncracked agglomerate at high magnification. Mainly gold particles were observed inside the agglomerate. It appears there was limited penetration of gangue particles like quartz due to absence of cracks. The presence of gold particle domination was probably due to its oleophilic properties and higher inertia due to superior density (19.5 
$\left.\mathrm{g} / \mathrm{cm}^{3}\right)$ compared to other metals $[17,18]$.

\section{Conclusions}

Generally the results from this work showed that pre-formed agglomerates developed from bio-diesel, castor oil and petroleum diesel as liquid hydrophobic phases can be used as effective material for recovery of liberated gold using bituminous coal from Kiwira as the solid hydrophobic phase.

Gold recovery rate can be increased by increase in agglomerate loading, surface area and the viscosity of the hydrophobic phase. Increase in gold particle concentration in the slurry increases recovery rate.

Prolonging contact time increased the capacity loading of agglomerates with possibility of selective gold penetration into coarse agglomerates making it possible to attain completion of gold recovery at lower agglomerate/ore ratios.

\section{Recommendations}

Following the good performance of the agglomerates used in the experiments it is recommended to carry out further work to test their performance using ores that contain variable gangue especially high sulphides ores rich in pyrites and arsenal pyrites, common in Tanzanian gold ores.

\section{References}

[1] A. Y. Ngenya, "Some Chemical Aspects of the Coal-Gold Agglomeration Process,” PhD Thesis, University of Dar es Salaam, 2004.

[2] R. D. Pehlke, "Unit Processes of Extractive metallurgy," Elsevier, North-Holland Publishing, 1977.

[3] R. W. Allen and T. D. Wheelock, "Effect of Preparation Techniques on Kinetics of Oil Agglomeration of Fine Coal," Minerals Engineering, Vol. 5, No. 6, 1992, pp. 649-660. doi:10.1016/0892-6875(92)90060-M

[4] J. Drzymala, T. D. Wheelock and R. W. Allen, "Basic Principles and Mechanisms of Selective Oil Agglomeration,” Pittsburgh Energy Technology Center, Pittsburgh, Pennsylvania, 1990.

[5] C. E. Capes and R. F. Germain, "Selective Oil Agglomeration in Fine Coal Beneficiation," Mineral Processing and Extractive Metallurgy Review, Vol. 2,
1982, p. 243.

[6] S. Gaidarjiev, "Coal-gold-Agglomeration of Alluvial Gold,” Fuel and Energy Abstracts, Vol. 38, No. 6, 1997, pp. 447-447.

[7] W. Kotze and F. W. Petersen, "Free Gold Recovery by Coal-Oil Agglomeration,” Journal South African IMM, Vol. 100, 2000.

[8] L. B. Moses and F. W. Petersen, "Flotation as Separation Technique in the Coal Gold Agglomeration Process," Minerals Engineering, Vol. 13, No. 3, 2000, pp. 255-264. doi:10.1016/S0892-6875(00)00005-4

[9] S. Sen, A. Seyrankaya and Y. Cilingir, "Coal-Oil Assisted Flotation for Gold Recovery,” Minerals Engineering, Vol. 18, No. 11, 2005, pp. 1086-1092. doi:10.1016/j.mineng.2005.03.007

[10] X. Q. Wu, R. J. Gochin and A. J. Monhemius, “Adhesion of Gold to Oil-Carbon Agglomerates,” Journal of Minerals Engineering, Vol. 17, No.1, 2004, pp. 33-38. doi:10.1016/j.mineng.2003.10.007

[11] A. Marciano, L. Costa and F. Lins, "Utilization of Coal-Oil Agglomerates to Recover Gold Particles,” Minerals Engineering, Vol.7, No. 11, 1994, pp. 1401-1409. doi:10.1016/0892-6875(94)90015-

[12] H. Gavin and A. J. Monhemius, “Improving Environmental, Economic and Ethical Performance in the Mining Industry,” Journal of Cleaner Production, Vol. 14, No. 12-13, 2006, pp. 1158-1167.

[13] J. R. Ikingura and M. K. Mutakyahwa, "Sources of Mercury Contamination and Exposure in Tanzania,” International Conference on Mining and Environment in Eastern and Southern Africa, SAREC and University of Dar es Salaam, October 1995.

[14] A. E. Mlaki, J. H. Katima and H. T. Kimweri, paper submitted for publication; Tanzania Journal of Engineering and Technology, 2010.

[15] M. Elblbesy and A. Hereba, "Effect of Viscosity and Surface Tension on Adhesion,” Current Applied Physics, Vol. 9, No. 4, 2009, pp. 872-874. doi:10.1016/j.cap.2008.08.006

[16] J. P. Calvez, M. J. Kim, P. L. Wong and T. Tran, "Use of Coal-Oil Agglomerates for Particulate Gold Recovery," Minerals Engineering, Vol. 11, No. 9, 1998, pp. 803-812. doi:10.1016/S0892-6875(98)00067-3

[17] T. L. Brown and H. E. Lemay Jr, "Chemistry: The Central Science,” Prentice Hall, Inc., New Jersey, 1985.

[18] A. Santine, (2006) Gold Jewelry publications. Available at: http://goldprice.org/gold-jewelry/2006/01/gold-jewelry-w eight.html 